\title{
Practical Measurement of X-ray Detection Performance of Large-Angle Silicon Drift Detectors Toward Quantitative Analysis in the Newly Developed 300 kV Aberration-Corrected Grand ARM
}

\author{
M. Watanabe*, T. Sasaki**, Y. Jimbo**, E. Okunishi**, H. Sawada** \\ * Dept of Materials Science and Engineering, Lehigh University, Bethlehem. PA 18015. \\ ** JEOL Ltd., 3-1-2 Musashino, Akishima, Tokyo, 196-8558, Japan.
}

X-ray analysis in (scanning) transmission electron microscopes (S/TEMs) has been rather limited due to the miserable signal availability in comparison with electron energy-loss spectrometry (EELS). However, X-ray analysis is brought back into the attention because of relatively simple nature and robust aspects, especially after the aberration-corrected STEM instruments become available. Additionally, the recent silicon drift detector (SDD) technology offers more flexible detector-instrument configurations, so that the limited availability of X-ray signals can be improved significantly. The latest aberration-corrected 300-kV JEOL Grand ARM instrument has been developed to achieve the best image resolution and analytical spatial resolution. The Grand ARM is equipped with two large solid angle SDDs: SDD1 is at the traditional geometry (perpendicular to the specimen-holder rod axis) with the take-off angle of 25 degree and SDD2 is placed at the new configuration (along with the holder rod axis) with the take-off angle of 29 degree. The pole-piece is designed to maximize X-ray collection by positioning 2 SDDs as close as possible to the optical axis. In this study, X-ray collection performance of 2 SDD systems was systematically measured and atomic-resolution X-ray analysis was also performed.

For measurement of X-ray detection performance, the NiOx thin film was used [1]. Figure 1 compares two X-ray spectra from the NiOx film measured by the two SDDs in the Grand ARM at $300 \mathrm{kV}$. The SDD2 with the new geometrical configuration shows better collection efficiency. By comparing X-ray spectra obtained from the previously calibrated HB 603 STEM, the collection angles of two detectors were determined. The conventional geometry SDD1 exhibits $0.593 \mathrm{sr}$ whereas the new configuration SDD2 shows $0.994 \mathrm{sr}$. When two SDDs are in the Grand ARM, X-ray signals can collected at the solid angle of $0.5 \pi \mathrm{sr}$, which is equivalent to $25 \%$ of the half sphere above the specimen. Using the NiOx film, the peak-to-background $(\mathrm{P} / \mathrm{B})$ ratio in Fiori definition and inverse hole count (IHC) of two SDDs were also measured in the Grand ARM and are compared with those in $300 \mathrm{kV} \mathrm{HB} 603$ and $200 \mathrm{kV}$ aberration-corrected STEMs (Fig. 2). Although the $\mathrm{P} / \mathrm{B}$ ratio of the both SDDs in the Grand ARM is comparable to that in other aberration-corrected instruments, the IHC values are superior to those in other instruments. Figure 3 summarizes the holder tilt-angle dependence of the $\mathrm{P} / \mathrm{B}$ ratio measured in ARM. It should be noted that the positive $\mathrm{x}$-tilt is toward the SDD1 and the positive $y$-tilt is towards the SDD2. The $\mathrm{P} / \mathrm{B}$ ratios plotted against the $\mathrm{x}$-tilt and $\mathrm{y}$-tilt are shown in Fig. 3(a) and (b), respectively. Fig. 3(c) shows the $\mathrm{P} / \mathrm{B}$ as a function of both $\mathrm{x}$ and $\mathrm{y}$ tilts, which means that the specimen is tilted between the both SDDs in positive direction. The SDD2 exhibits lower P/B values than the SDD1 but less sensitive to the specimen tilt angle. Although the P/B values in the SDD2 is slightly degraded, the SDD2 shows higher solid angle than the SDD1, which implies that this new geometry can improve X-ray collection efficiency.

A set of X-ray maps obtained from $\mathrm{SrTiO}_{3}$ using the Grand ARM is shown in Fig. 4. The maps with $256 \times 256$ pixels were acquired only for $2.5 \mathrm{~min}$ in total with the probe current of $80 \mathrm{pA}$. 
These atomic-resolution X-ray maps can be acquired with the limited current for very short acquisition time, which can be beneficial for characterization of irradiation sensitive materials.

\section{References}

[1] R.F. Egerton \& S.C. Cheng, Ultramicrosc. 55 (1994), 43.

[2] The author (MW) wishes to acknowledge financial support from the NSF through grants DMR-0804528 and DMR-1040229.

Fig. 1

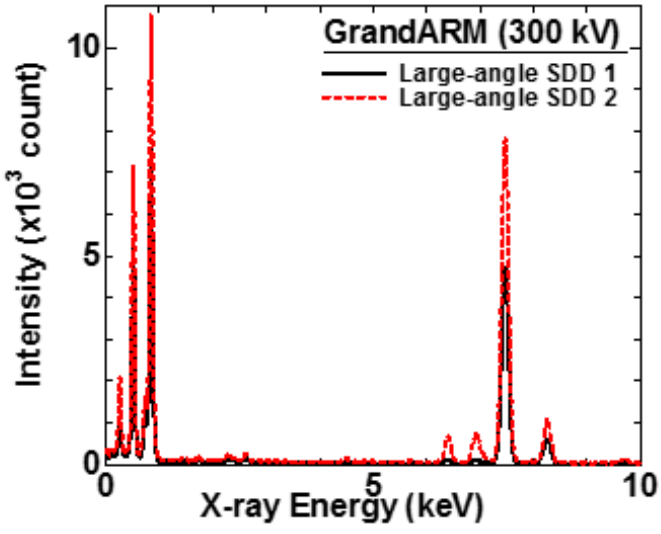

Fig. 3
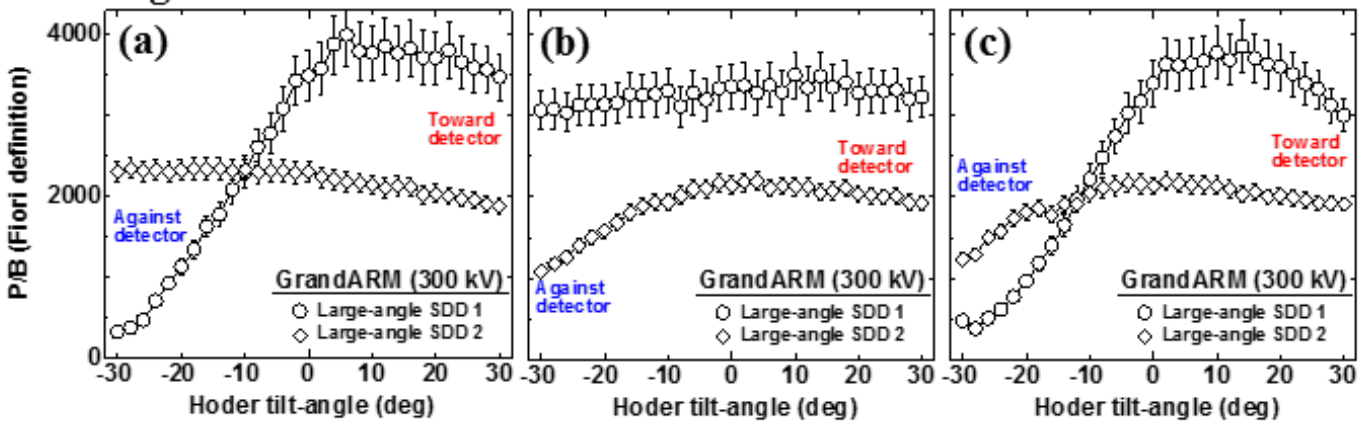

Fig. 4
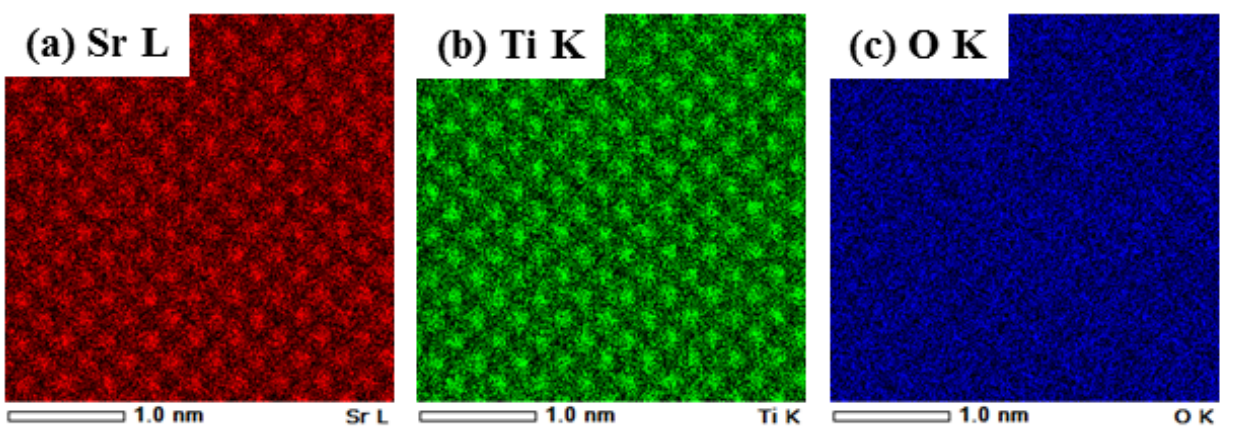

Figure 1: X-ray spectra from NiOx measured by two SDDs in Grand ARM.

Figure 2: P/B plotted against IHC measured in different instruments, including Grand ARM.

Figure 3: Tilt angle dependence of P/B measured in Grand ARM: (a) x-, (b) y-and (c) x/y-tilts.

Figure 4: Atomic-resolution X-ray maps measured from $\mathrm{SrTiO}_{3}$ in Grand ARM. 